\title{
Smart Alignment Tool for Knee MosaicPlasty Surgery
}

\author{
Albert W. Brzeczko ${ }^{1}$, Randal P. Goldberg ${ }^{2}$, Russell H. Taylor ${ }^{2,3}$, Peter Evans ${ }^{4}$ \\ 1 Baltimore Polytechnic Institute High School, Baltimore, MD, USA \\ 2 Mechanical Engineering Department, The Johns Hopkins University, Baltimore, MD, \\ USA \\ 3 Computer Science Department, The Johns Hopkins University, Baltimore, MD, USA \\ 4 Department of Orthopedic Surgery, Johns Hopkins Hospital, Baltimore, MD, USA
}

\begin{abstract}
The Smart Alignment Tool for Knee MosaicPlasty Surgery is a device that aids in aligning the MosaicPlasty harvesting chisel with the cartilage surface of the knee. In the standard arthroscopic procedure, the angle at which the graft is harvested and inserted is determined visually by the surgeon through a single endoscopic view. The ability of the surgeon to obtain and place these grafts in an orientation perpendicular to the cartilage surface determines, in large part, the efficacy of the procedure. By instrumenting the tool, the angle of the tool relative to the surface is fed back to the surgeon. Preliminary experiments show this greatly increases the accuracy and consistency of this task.
\end{abstract}

\section{Introduction}

The MosaicPlasty procedure for knee osteochondral grafts, pioneered by Dr. Lazló Hangody describes a technique to repair articular cartilage degeneration onset by

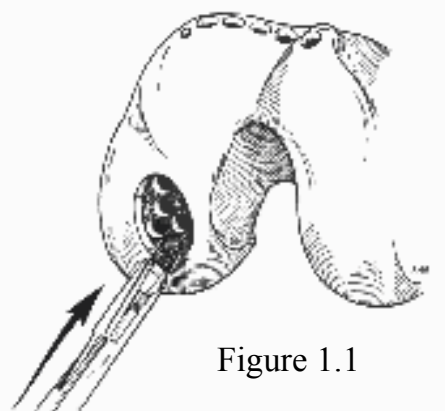
trauma or aging (1). The defects in the cartilage are replaced by cylindrical osteochondral grafts taken from a non-weight-bearing portion of the knee, such as the patellofemoral joint. The grafts fill the sections of the knee where the cartilage had been worn (Figure 1.1). Because these grafts are smaller than other autograft transplantation procedures, and because the procedure integrates transplanted cartilage with the adjacent hyaline cartilage via fibrocartilage, MosaicPlasty provides a smooth cartilage surface, mimicking closely the surface of the knee before it had suffered from defects. Another advantage of the MosaicPlasty procedure is the integration of the spongy element of the graft, which fuses with the spongy bed at the recipient site (2). The resulting cartilage surface is very stable because it has a strong foundation with the underlying bone. 
A procedural aspect that greatly impacts the efficacy of the MosaicPlasty procedure is the harvesting and placement of the osteochondral graft. If the graft is not harvested and placed perpendicular to this surface, many of the advantages of the technique are lost, as the implanted cartilage does not line up properly with the surface. Additionally, the projecting element of the cartilage has very little support and behaves as cartilage placed on an osseous surface (2). The possibility also exists that the articular surface may be injured by the misaligned graft-harvesting tool (3). Presently when performing this procedure arthroscopically, the surgeon looks through an endoscope to estimate the perpendicularity of the chisel, a difficult task to perform from the single view present. This difficulty causes a majority of these procedures to be performed open.

The Smart Alignment Tool, an angle-sensing device that mounts on the MosaicPlasty graft-harvesting tool, is able to detect the angle between the bone surface and a vertical reference axis. The device uses three points, maintained by feelers placed on the cartilage surface and tracked by linear potentiometers, to locate the cartilage surface, and compare its normal to the axis of the tool. This gives a quantitative measurement of the vital angle independent of the surgeon's ability to perform visual inspection.

\section{Prototype Design}

\subsection{Requirements}

The first step of the project was determining the constraints the MosaicPlasty surgery would pose on the design. The device needed to fit within an arthroscopic portal, so size was a very important consideration. Ideally, the device's diameter should be within approximately $5 \mathrm{~mm}$ that of the grafting chisel so the size of the portal needed would not need to be increased significantly. The device should also allow the chisel to be hammered in approximately $15 \mathrm{~mm}$ to the osteochondral surface. The device should provide angles accurate to approximately $1^{\circ}$. In order for the Smart Alignment Tool to become a useful surgical tool, it must be completely self-contained, including onboard signal processing and feedback display hardware. Finally, the device should be durable enough to withstand sterilization and the force of hammering the chisel.

\subsection{Design}

It was determined that the design of the Smart Alignment Tool prototype would be based on an $8.5 \mathrm{~mm}$ chisel, as this was the largest available size, and thus the easiest to build upon. The use of this size required that the total outer dimension of the Smart Alignment Tool be approximately $14 \mathrm{~mm}$ or less.

The device uses three "feelers" to contact the cartilage surface. These feelers are permitted to slide parallel to the axis of the tool as it is oriented, maintaining the surface of the knee. Motion of these feelers is tracked with BI Technologies Model 404 linear compact potentiometers with spring-returning sliders of approximately 
$15 \mathrm{~mm}$ travel length, and midrange resistance values $(\sim 10 \mathrm{k} \Omega)$. Each potentiometer returns a voltage that corresponds to the feeler position.

The grafting chisel is rigidly mounted within the Smart Alignment Tool, locked in place with a setscrew. The potentiometers allow sufficient range of motion for the tool alignment and insertion to be performed.

\subsection{Signal Processing}

The next task was to create a data acquisition (DAQ) system for the tool so that the voltages output from the potentiometers could be processed into an angle. As a preliminary setup, a National Instruments Lab PC+ ISA DAQ card and LabVIEW software were used. However, this PC-dependent setup went against the requirement for the device to be as portable as possible. It was necessary to implement secondary signal processing setup allowing the Smart Alignment Tool to be free of a full-sized computer. The Handy Board, a C-programmable Motorola 68HC11-based controller board designed by MIT, is being used as an intermediate step.
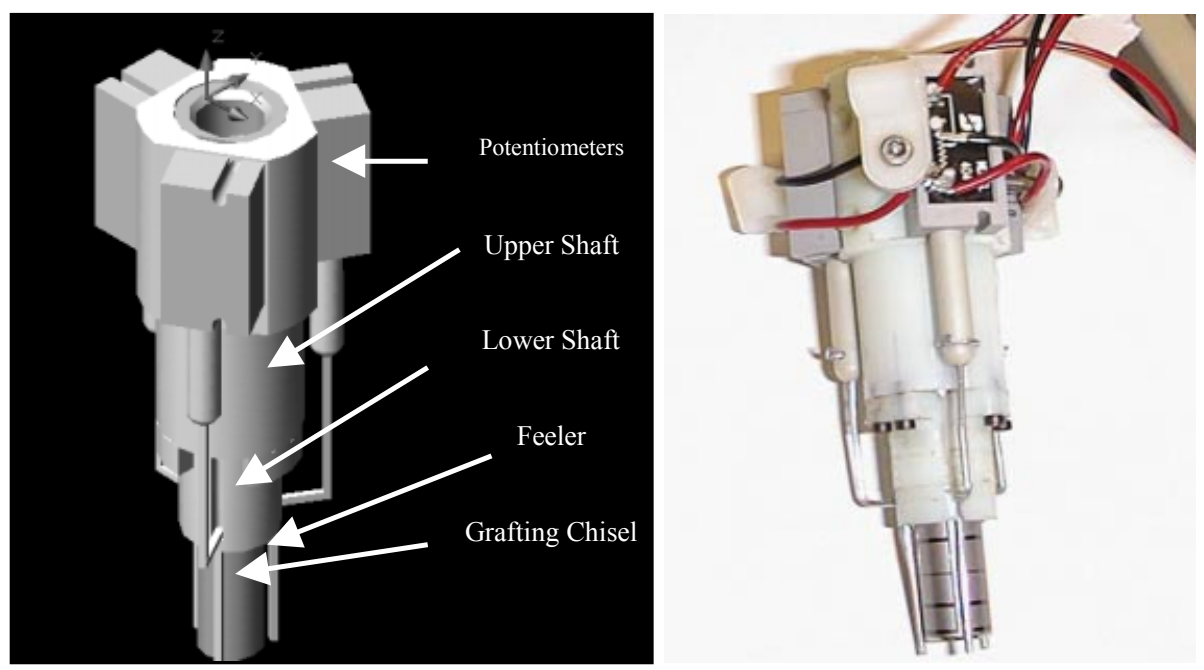

Figure 2.1: CAD Model and Photo of Prototype Alignment Tool

A program was written for the Handy Board that converts each potentiometer voltage output into a Z-coordinate (parallel to the axis of the tool). The X-and Ycoordinates are fixed per the construction of the device. The three resulting points define the surface of the cartilage. The normal of this surface is then compared to the $\mathrm{Z}$-axis of the tool to obtain a measurement of the angle between them. This angle is then output to the Handy Board's LCD for viewing. 


\section{Experiments}

To begin with, a simple validation experiment was performed. A calibrated Optotrack Rigid Body was mounted in the Smart Alignment Tool, and the angle measured by the Smart Alignment Tool was compared to the Optotrack reading. The experiment showed that the device was accurate to an average accuracy of $1.39^{\circ}$.

Next, two sets of experiments simulating the arthroscopic surgery were performed. In the first set of experiments, the operator's field of vision and range of motion were impaired in ways resembling the actual MosaicPlasty procedure. A box enclosing a
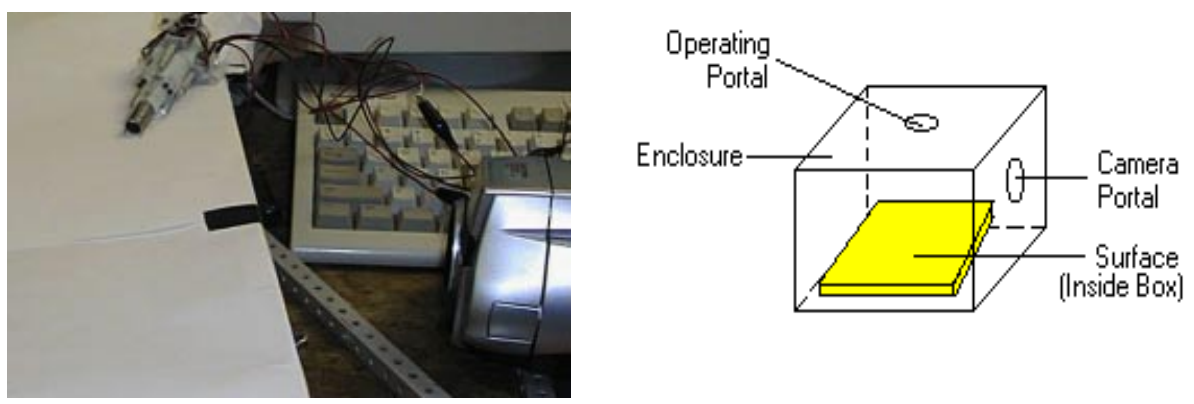

Figure 3.1: Diagram and Photo of Experimental Setup

flat surface at an arbitrary angle to the table was built, with access and view portals to mimic the endoscopy (Figure 3.1). The grafting chisel and inactive Smart Alignment Tool were placed in the access portal and a camera was placed in the vision portal. Eleven different subjects then performed five timed trials in which they attempted to place the chisel perpendicular to the surface, relying solely on eyesight. After chisel placement, the Smart Alignment Tool was activated and the angle between chisel placement and normal to the surface was recorded.

In the second set of experiments, the Smart Alignment Tool was active the entire time. Test subjects were thus given visual information from the camera and angle readouts from the Smart Alignment Tool. As was the case in the first experiment, five trials were conducted. After each chisel placement, elapsed time and the placement angle were recorded.

From these experiments, the time and accuracy of chisel placement without the aid of the Digitizer was compared to the time and accuracy with the Digitizer.

\section{Results}

Results of experiments were as follows. "Angle" denotes degrees divergence from perpendicularity to the test plane. "Time to place" denotes the elapsed time before perpendicularity was perceived and test instrument was driven. 
4.1 Results of Experiment without Active Smart Alignment Tool:

\begin{tabular}{|c|c|c|c|c|}
\hline \multirow{2}{*}{ Subject } & \multicolumn{2}{|c|}{$\begin{array}{c}\text { Angle Placement } \\
\text { (Degrees) }\end{array}$} & \multicolumn{2}{c|}{ Time Elapsed (seconds) } \\
\cline { 2 - 5 } & Mean & $\begin{array}{c}\text { Standard } \\
\text { Deviation }\end{array}$ & Mean & $\begin{array}{c}\text { Standard } \\
\text { Deviation }\end{array}$ \\
\hline 1 & 2.6 & 1.1 & 21.8 & 12.5 \\
\hline 2 & 3.2 & 1.6 & 11.0 & 2.5 \\
\hline 3 & 5.8 & 1.8 & 29.4 & 14.5 \\
\hline 4 & 9.8 & 3.4 & 30.0 & 9.4 \\
\hline 5 & 3.4 & 2.1 & 8.6 & 4.6 \\
\hline 6 & 11.8 & 7.4 & 6.2 & 1.8 \\
\hline 7 & 6.0 & 5.7 & 13.0 & 13.3 \\
\hline 8 & 7.8 & 3.8 & 14.8 & 8.8 \\
\hline 9 & 8.0 & 3.7 & 16.0 & 11.8 \\
\hline 10 & 11.4 & 2.7 & 14.6 & 5.5 \\
\hline 11 & 5.2 & 2.5 & 14.8 & 4.1 \\
\hline Overall & 6.8 & 4.5 & 16.4 & 11.0 \\
\hline
\end{tabular}

\subsection{Results of Experiment with Active Smart Alignment Tool:}

\begin{tabular}{|c|c|c|c|c|}
\hline \multirow{2}{*}{ Subject } & \multicolumn{2}{|c|}{$\begin{array}{c}\text { Angle Placement } \\
\text { (Degrees) }\end{array}$} & \multicolumn{2}{c|}{$\begin{array}{c}\text { Time Elapsed } \\
\text { (Seconds) }\end{array}$} \\
\cline { 2 - 5 } & Mean & $\begin{array}{c}\text { Standard } \\
\text { Deviation }\end{array}$ & Mean & $\begin{array}{c}\text { Standard } \\
\text { Deviation }\end{array}$ \\
\hline 1 & 0.0 & 0 & 40.0 & 19.8 \\
\hline 2 & 1.4 & 1.1 & 8.4 & 7.6 \\
\hline 3 & 0.6 & 0.5 & 37.2 & 32.9 \\
\hline 4 & 1.2 & 1.6 & 22.6 & 9.8 \\
\hline 5 & 0.8 & 0.4 & 10.8 & 7.6 \\
\hline 6 & 1.4 & 0.9 & 9.8 & 6.7 \\
\hline 7 & 1.0 & 0.9 & 14.0 & 6.5 \\
\hline 8 & 0.4 & 0.5 & 34.8 & 16.3 \\
\hline 9 & 0.4 & 0.5 & 17.8 & 18.1 \\
\hline 10 & 3.2 & 2.5 & 14.4 & 3.9 \\
\hline 11 & 0.6 & 0.5 & 21.2 & 6.4 \\
\hline Overall & 1.0 & 1.3 & 21.0 & 17.2 \\
\hline
\end{tabular}

\section{Discussion}

\subsection{Analysis of Results}

The data show that the chisel was placed more accurately and with greater consistency when using the Smart Alignment Tool for feedback. The mean angle 
from the normal was $1.0^{\circ}$ with the aid of the Smart Alignment tool and $6.8^{\circ}$ without it, including maximum angular error of $20^{\circ}$. The mean elapsed time increased in the Smart Alignment Tool trials. However, this can be attributed to the fact that the subjects had numeric angle readouts, and were willing to take extra time to ensure the angle was placed accurately. For the subjects using only eyesight, the overall standard deviation of angle placement was 4.5 , versus 1.3 with the Smart Alignment Tool's angle readout.

It should be noted that the validation experiments indicated an average error of $1.4^{\circ}$ and maximum errors of up to $3.4^{\circ}$ after four outliers were removed due to drastic bending of the feelers. While this shows that the device is less accurate than the target $1^{\circ}$ error, it does not change the qualitative conclusions that can be drawn from the simulated surgery experiments. Rather, it points at clear improvements to be made in forthcoming iterations of the design.

\subsection{Future Plans/Experiments}

A problem with the current prototype arises from the stiffness of the feelers, stemming from the feeler material and wear in the plastic body by which they are guided. In the next iteration of the Smart Alignment Tool, these features will be made of stiffer material, which will significantly improve the accuracy of the device.

Another feature to be added is a "footprint". The current feeler tips are likely to pierce the cartilage surface, rather than rest on them. In the next iteration, a ring will be mounted to the bottoms of the three feelers, increasing their footprint and distributing their force more evenly along the cartilage surface. This will further aid in stiffening the feelers, as well as reduce errors which may be introduced by the unevenness in the cartilage surface.

Other design refinements will be made to increase the overall functionality of the device. An onboard signal processor would contribute to the compactness and portability of the Smart Alignment Tool to directly display the angle readout. With this comes the ability to display additional information. Work is being done to determine the most effective way to indicate the direction and magnitude of the angle on such a display. From observation in the experiments, this extra information would likely have a significant positive impact on time it takes to align the tool. It will also be advantageous to modify the design such that the Smart Alignment Tool can accommodate a full range of chisel sizes.

Ultimately, cadaver experiments and clinical trials will be performed to further demonstrate the Smart Alignment Tool's effect on the results of the Knee MosaicPlasty procedure.

\section{Acknowledgments}

The authors gratefully acknowledge the support of the National Science Foundation Engineering Research Center Grant \#EEC9731478. 


\section{References}

1) Bobic, Vladimir. The Utilisation of Osteochondral Autografts in the Treatment of Articular Cartilage Lesions. 15 Sep. $2000<\mathrm{http} / / / w w w . i s a k o s . c o m / i n n o v a t i o n s / o a t s . h t m l>$.

2) Christel, P., et al. Osteochondral Grafting using the Mosaicplasty Technique. 15 Sep. 2000 $<$ http://www.maitriseorthop.com/corpusmaitri/orthopaedic/mo76_mosaicplasty/index.shtml>.

3) Higgs, Geoffrey B, and Arthur L Boland. Cartilage Regeneration and Repair. 15 Sep. 2000 $<$ http://www.mgh.harvard.edu/depts/hoj/html/cartilage_repair.html $>$. 